\title{
Computational Modeling of Jet Induced Mixing of Cryogenic Propellants in Low-G
}

J. I. Hochstein and P. M. Gerhart

The University of Akron

Akron, Ohio

and

J. C. Aydelott

Lewis Research Center

Cleveland, Ohio

Prepared for the

Twentieth Joint Propulsion Conference

cosponsored by the AIAA, SAE, and ASME

Cincinnati, Ohio, June 11-13, 1984 

EMEF:

15

$1 \cdots \quad 1$ RU/NASA-TM- 33703

OISPLAY IO/6/1

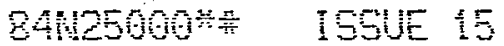

E-2161 MAS 1 . 1538303

PACE 2321 CATEOP 34 PFT

UWCLASIFIED DOCUMENT

AIAA-84-1344 OUI \#AG

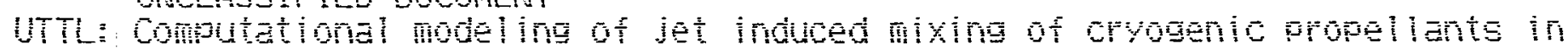
$10 \mathrm{ii}-\mathrm{G}$

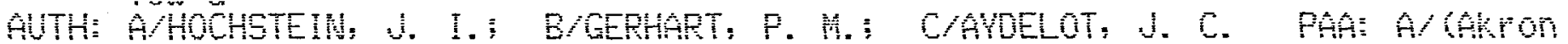
Univis Er(Akron Univ.)

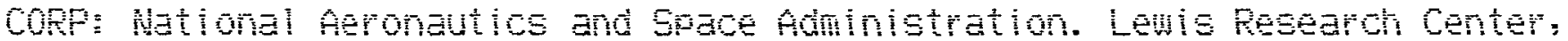

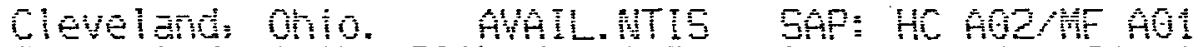

Fresented the 20 th jomt prombion Cont, Chomati, $11-13$ Jun. 1984; SPONSORE by AIAA, SAE and AGAE

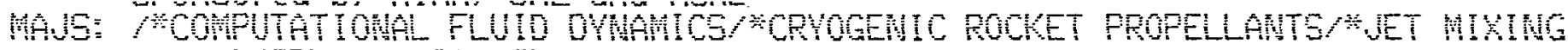
FLOW

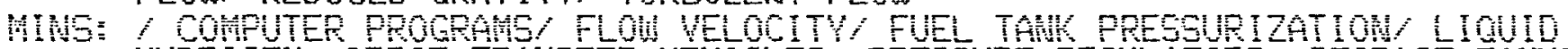

ABA: AutTor HOROGEN OREIT TRANGER VEHICLES PRESSURE REGULATORS STORAGE TANKS 


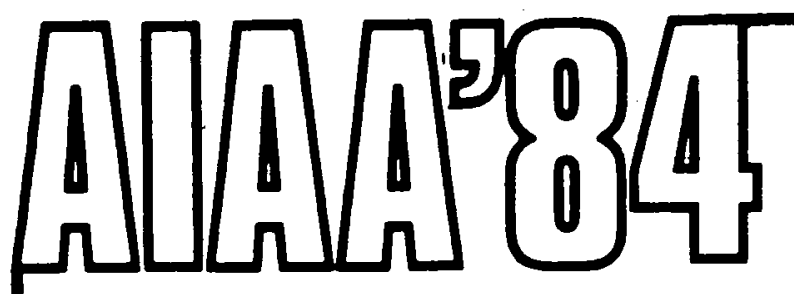

AIAA-84-1344

Computational Modeling of Jet Induced Mixing of Cryogenic Propellants in Low-G

J. I. Hochstein and P. M. Gerhart, The University of Akron, Akron, $\mathrm{OH}$; and J. C. Aydelott, NASA Lewis Research Center, Cleveland, $\mathrm{OH}$

\section{AIAA/SAE/ASME \\ 20th Joint Propulsion Conference \\ June 11-13, 1984/Cincinnati, Ohio}





\author{
J. I. Hochstein* and P. M. Gerhart** \\ The University of Akron \\ Akron, Ohio 44325 \\ J. C. Aydelott*** \\ National Aeronautics and Space Administration \\ Lewis Research Center \\ Cleveland, Ohio 44135
}

\section{Abstract}

The SOLA-ECLIPSE Code is being developed to enable computational prediction of jet induced mixing in cryogenic propellant tanks in a low-gravity environment. Velocity fields, predicted for scale model tanks, are presented which compare favorably with the available experimental data. A full scale liquid hydrogen tank for a typical Orbit Transfer Vehicle is analyzed with the conclusion that coupling an axial mixing jet with a thermodynamic vent system appears to be a viable concept for the control of tank pressure.

\section{Nomenclature}

$C_{\mu}$ - turbulence model constant $=0.09$

$C_{\varepsilon 1}$ - turbulence model constant $=1.44$

$C_{\varepsilon 2}$ - turbulence model constant $=1.92$

$C_{\varepsilon 3}$ - turbulence model constant $=0.79$

$\mathrm{G}_{\Phi}$ - generalized production term

$j$ - switching integer

$k \quad$ - turbulence kinetic energy

$P \quad$ - pressure

$S_{\Phi}$ - generalized destruction term

$t \quad$ - time

$u, v-x$ and $y$ velocity components respectively

$x, y$ - spatial coordinates, cartesian or cylindrical ( $x$ in radial direction)

$\Gamma_{\phi} \quad$ - generalized diffusion coefficient

$\varepsilon \quad$ - turbulence energy dissipation rate

$\mu_{\mathrm{t}}$ - "effective" viscosity

$\rho$ - density

$\sigma_{k} \quad$ - Prandt l-Schmidt number for $k, \sigma_{k}=1.0$

$\sigma_{\varepsilon} \quad$ - Prandt 1-Schmidt number for $\varepsilon, \sigma_{\varepsilon}=1.3$

$\Phi^{\varepsilon} \quad$ - generalized variable

\section{Introduction}

Investigations of liquid propellant behavior in a low-gravity environment have been managed and actively pursued by the NASA Lewis Research Center (LeRC) for over twenty years. The current emphasis of the program is directed towards the development of technology for the design of fluid management systems including liquid acquisition, thermal control of cryogenic tankage, and fluid transfer. The work being reported is focused on problems associated with cryogenic propellant tank pressure control.

* Special Lecturer, Mechanical Engineering.

** Professor, Mechanical Engineering.

*** Aerospace Engineer.
The motivation for examining the mixing of cryogenic propellants is a desire to efficiently employ a Thermodynamic Vent System (TVS) to control cryogenic propellant tank pressure. The problems associated with a simple direct vent system are documented in reference 1 . The TVS operates by withdrawing liquid from the tank, cooling it by expansion through a Joule-Thomson valve, circulating it through a heat exchanger, and finally venting it overboard. The propellant remaining in the tank is cooled by passing it through the heat exchanger and reinjecting it into the tank. The efficient application of this concept requires that the cooled propellant be well mixed with the the other liquid in the tank in order to keep all of the liquid below the boiling point.

Scale model experiments using typical propellant tank configurations have been performed in the LeRC Zero-G Facility. Reference 2 reports the results of experiments which used ethanol in plexiglass tanks to simulate the behavior of the propellants. Dyed ethanol was injected to simulate a mixing jet and high-speed photography was used to record the mixing. Ethanol was chosen as the experimental fluid because the use of actual cryogenic propellants was impractical. The experiments were performed in $10 \mathrm{~cm}$ diameter tanks because only five seconds of free fall is available for the experiment. In this size tank, approximately two seconds is required for the liquid-vapor interface to reach a zero-g configuration, leaving three seconds of experimental time to examine the liquid jet mixing phenomena. A dyed liquid jet was used due to the difficulty of establishing and measuring temperature gradients in small scale tanks with limited test time. The experimental results were used to develop flow characterization and mixing parameters which can be used to determine mixer requirements for full scale tanks.

Several factors provide motivation for developing the capability of computationally predicting these flows. A computational analysis is not restricted to small tanks, short times, or simulated propellants. It can predict both the velocity and temperature fields due to the injection of a cool jet. Further, it will provide a more economical tool for investigating design and operational paramaters.

The SOLA-ECLIPSE Code (Energy Calculations for LIquid Propellants in a Space Environment) is being developed to examine jet induced mixing of cryogenic propellants in a low-g environment. The starting point for its development was the NASA SOLA-VOF Code ${ }^{3}$ which was written to analyze the draining of cryogenic propellants from tanks in 
low-g. The following sections present a brief description of the development of SOLA-ECLIPSE, the results which have been obtained by modelling the drop tower experiments 4 , and preliminary results for typical Orbit Transfer Vehicle (OTV) liquid hydrogen tanks.

\section{Outline of NASA-SOLA-VOF}

The NASA SOLA-VOF Code is a derivative of the SOLA family of codes which use the SOLA algorithm to solve the two-dimensional (Cartesian or cylindrical) Navier-Stokes equations on an Eulerian mesh of rectangular computational cells. The cell size may be varied by row and by column. The code solves the laminar hydrodynamic problem for the primitive variables $P, u$, and $v$. A staggered grid is used with velocities evaluated at cell faces and pressure defined at cell centers.

The VOF (Volume-of-Fluid) concept is based on a function, $F$, which has a value of one at any point in space occupied by fluid and a value of zero at a point which is not occupied by fluid. With $F$ so defined, the fractional volume of a cell which contains fluid is the average value of $F$ for that cell. Cells through which a free surface passes are identified by values of $F$ between zero and one. The free surface location and shape is determined by evaluating $F$ in the cell being considered and in its neighbors. The curvature of the surface is computed, and surface tension effects are modeled by imposing an appropriate pressure at the free surface.

\section{Turbulence Model}

The NASA SOLA-VOF code solves the laminar hydrodynamic problem. Since the goal of injecting the jet into the tank is to induce mixing, it is anticipated that a turbulent jet will be used due to its superior mixing characteristics as compared to a laminar jet. The following paragraphs describe the turbulence model which was installed in SOLA-ECLIPSE.

The $k-\varepsilon$ turbulence model was chosen as the turbulence model for the code because it is the simplest model which has a good probability of succcessfully modeling the recirculating flow which is induced by the mixing jet. It is the most widely used two equation turbulence model and its success with flows of this type is well documented ${ }^{5}$. This model results in the computation of an "effective viscosity" which is then used in the momentum equations. The two differential equations of the $k-\varepsilon$ model, and the equation to compute the "effective" viscosity, can be written in the following form for two dimensional incompressible flow:

$$
\begin{gathered}
\rho \frac{D \Phi}{D t}=\frac{1}{x^{j}} \frac{\partial}{\partial x}\left(x^{j} \Gamma_{\Phi} \frac{\partial \Phi}{\partial x}\right)+\frac{\partial}{\partial y}\left(\Gamma_{\Phi} \frac{\partial \Phi}{\partial y}\right)+G_{\Phi}+S_{\Phi} \\
\text { where: } \Phi=k, \varepsilon \\
j=0-\text { Cartesian } \\
1-\text { cylindrical } \\
\quad \\
\mu_{t}=C_{\mu} \rho \frac{k^{2}}{\varepsilon}
\end{gathered}
$$

k-equations

$\Gamma_{k}=\frac{\mu_{t}}{\sigma_{k}}$

where:

$$
G_{k}=\mu_{t} B
$$

$$
\begin{aligned}
B=2\left[\left(\frac{\partial u}{\partial x}\right)^{2}+\left(\frac{\partial v}{\partial y}\right)^{2}+j\left(\frac{u}{x}\right)^{2}\right]+\left(\frac{\partial u}{\partial y}+\frac{\partial v}{\partial x}\right)^{2} \\
S_{k}=\rho \varepsilon \\
\varepsilon \text {-equations } \\
\Gamma_{\varepsilon}=\frac{\mu_{t}}{\sigma_{\varepsilon}} \\
G_{\varepsilon}=C_{\varepsilon 1} \mu_{t} B \frac{\varepsilon}{k} \\
S_{\varepsilon}=C_{\varepsilon 2^{\rho} \frac{\varepsilon^{2}}{k}}
\end{aligned}
$$

Although this set of expressions and values has produced good results for plane jets and for wall boundary layers, it overpredicts the spread rate of axisymmetric jets by approximately forty percent. Several functional dependencies for the constants have been proposed to cure this problem $6,7,8$, but all the proposed relationships require computations involving jet width and/or centerline velocity gradient which would greatly complicate computations. Reference 9 proposes that the existing constants and expressions be retained with the inclusion of the following expression as an additional source term in the $\varepsilon$-equation.

$$
C_{\varepsilon 3} \rho \frac{k^{2}}{\varepsilon} \cdot \frac{1}{4}\left(\frac{\partial v}{\partial x}-\frac{\partial u}{\partial y}\right)^{2} \cdot \frac{u}{x}
$$

This term accounts for the vortex stretching which occurs in axisymmetric jets but is not present in true 2-D flows. The value of $C_{E} 3$ was chosen by the author of reference 9 to produce the correct spread rate for axisymmetric jets.

The question of appropriate solid boundary conditions for this turbulence model has been addressed by many workers. A law-of-the-wall boundary condition has been installed in SOLA-ECLIPSE in order to avoid the cell packing required to resolve the steep gradients near a wall. This approach replaces the computations of the gradients by approriate expressions derived from a law-of-the-wall analysis. The actual expressions used in the code are developed in reference 10 .

The free surface boundary conditions for the turbulence model were chosen to be as simple as possible since the free surface activity is not the focus of this study. Since turbulence aids the mixing process, underpredicting turbulence will result in conservative results. This can be achieved by underpredicting the turbulence kinetic energy and overpredicting the turbulence energy dissipation rate. This is accomplished in the code by setting $k$ equal to zero in a surface cell and setting $\varepsilon$ equal to the value of $\varepsilon$ in the 
adjacent full cell. Although no claim is made that the code accurately models turbulence effects at the free surface, the accuracy of the results obtained for the bulk fluid behavior should not be significantly affected.

\section{Computational Results for Model Tanks}

As previously described, experiments have been performed to examine jet induced mixing in zero-g by injecting a dyed jet into partially filled scale model tanks. The first cases to be modeled with SOLA-ECLIPSE were replications of experimental conditions. The following paragraphs discuss the predicted velocity fields for one laminar jet and for one turbulent jet for which experimental data is available.

The computational mesh used to analyze the scale model tanks is shown in Figure 1 and the resulting code approximation to the tank is shown in Figure 2. The variable mesh capability of SOLA-ECLIPSE has been used to refine the mesh in regions which are expected to contain sharp gradients. The vector field displays show both the true curve of the tank head and the stairstep approximation to it. For both of the cases being presented, the upper head has been removed since no flow occurs in this region. A scaling vector is supplied so that vector magnitudes can be interpreted. The experimental tanks are $10 \mathrm{~cm}$ in diameter and the scaling velocity is given in $\mathrm{cm} / \mathrm{sec}$. Velocities smailer than two percent of the jet velocity are not displayed. Fluid is injected through the $0.4 \mathrm{~cm}$ diameter pipe which penetrates the lower head and is simultaneously withdrawn through the annulus surrounding the pipe. The heading includes the elapsed time from jet initiation (seconds) and the number of cycles (time steps) required by the code to reach this point in the transient. The detail of the free surface shape very close to the wall is misrepresented by the graphics but is accurately represented in the calculation procedure. The two scale model cases have been run to an elapsed time of three seconds which was the maximum time available for mixing in the LeRC Zero-G Facility. The development of the laminar jet is

depicted at one second intervals in Figures 3, 4, and 5. The highly curved free surface is a reflection of the zero-g environment where surface tension is an important force. The jet Reynolds number for this flow is 450 which is well below the transition value. The experimental data 4 revealed no measurable spreading of this jet. Comparison between the predicted velocity field and the experimental results is clouded by the inherent differences in information being evaluated. The experimental data consists of pictures of dye spreading which really represents the concentration field being produced. Hence, the picture only reveals the current location of fluid which was injected. In contrast, the predicted velocity field shows both the motion of fluid which originated in the pipe and the induced flow of the fluid which initially resided in the tank. It is therefore necessary to estimate where the edge of the jet indicated by dye diffusion would lie in the predicted velocity field.

Examination of the predicted velocity field shows very little induced flow and leads to the inference of very little jet spreading. Figure 3 shows a slight mushrooming of the head of the jet.
This is characteristic of the the jet development prior to reaching the free surface and is in agreement with the experimental data. Figures 4 and 5 show a slight bulge in the free surface at the centerline of the tank. Although the bulge is more gradual and of a smaller height than the experimental results, the difference is not large and it does not change the fundamental nature of the flow. Examination of the velocity field after three seconds of jet operation indicates approximately the same spread of jet fluid along the surface toward the tank walls as was found in the experiment.

The turbulent jet case modeled with the code used the same physical configuration as the laminar case. The jet velocity is $50.1 \mathrm{~cm} / \mathrm{sec}$ which corresponds to a jet Reynolds number of 1320. The predicted velocity fields are shown in Figures 6,7 , and 8 . An acceleration of $0.0022 \mathrm{~g}$ 's was specified to match the experimental conditions. As can be seen by comparison whith the laminar case which was in a zero-g environment, even a very small acceleration will have a significant impact on the curvature of the free surface. Examination of the predicted velocity fields reveals several interesting features. First, the induced velocity field is much more extensive than was predicted for the laminar jet. Although the edge of the jet is again difficult to determine, a ten degree spread rate appears reasonable based on the velocities displayed. This is in good agreement with both theoretical predictions for steady axisymmetric jets and with the experimental results ${ }^{4}$. The centerline bulge is again underpredicted but the character of the experimental and predicted flows is the same. The experiment shows dye spreading all the way to the tank walls and starting to flow back toward the tank head. The field displayed in Figure 8 appears to be in agreement with this level of development. Figure 6 shows considerable free surface activity, even before the jet reaches the free surface. In order to compare the predicted level of activity to the experimentaliy observed activity, a movie film was made by sequentially displaying predicted velocity fields. Comparison of the computer generated $f i l m$ and the experimental data film shows comparable levels of free surface activity.

\section{Orbit Transfer Vehicle Tank}

The first full scale tank to be modeled was a liquid hydrogen tank for a typical Manned Orbit Transfer Vehicle (OTV) 11 . The tank is slightly more than two meters in radius and the cylindrical section has a length of almost six meters. Although the proposed tank has elliptical heads, the code does not currently have the capability of modeling elliptical heads therefore hemispherical heads were used for the computational model. The computational mesh used for analysis of this case is shown in Figure 9. The tank was assumed to be fifty percent full in a zero-g environment which resulted in the initial conditions depicted in Figure 10. The correlations developed in reference 4 were used to select a jet velocity which would result in a deformation of the free surface but would not result in a large geyser. A tank-to-jet radius ratio of $22: 1$ was chosen to approximate the ratio of the experimantal tanks of the previous section. A velocity of $4.51 \mathrm{~cm} / \mathrm{sec}$ 
was chosen which corresponds to a jet Reynolds number of 50,000 . The code was run to an elapsed time of two hours. It was felt that this was sufficient time to determine the feasability of using a mixing jet in conjunction with a TVS to cool the bulk of the propellant in this configuration.

The development of the velocity field predicted by SOLA-ECLIPSE for this case is shown in Figures $11,12,13$, and 14. After five minutes of operation, the jet has reached the free surface and is spreading radially. The predicted field after twenty minutes of jet operation shows that at least a small velocity has been induced in most of the fluid. Comparison of the predicted fields after one and two hours indicates that the velocity field is fully developed after one hour. Almost all of the fluid in the tank has been set in motion. After one hour, the large recirculation region which was anticipated has appeared and extends from the free surface down into the upper part of the lower head. These results can be compared to the correlations for geyser height and for mixing time developed in reference 2 . The correlation predicts a geyser height of 0.4 tank radii whereas SOLA-ECLIPSE predicts a geyser height of approximately 0.1 tank radii. Although agreement on geyser height seems poor, the character of the mixing is not significantly different for the two predictions. In both analyses the jet does not pierce the free surface but is deflected toward the tank walls. The mixing time correlation predicts the time required to completely mix the fluid in the tank. For the situation being analyzed, the correlation predicts a mixing time of 3700 seconds. Since the velocity field predicted by SOLA-ECLIPSE appears to have reached steady state by this time, the conclusion of thorough mixing seems reasonable.

A word of caution must be voiced concerning the conclusions which can be drawn from these results. The purpose of the jet is to disperse the cooling effect of a TVS in order to subcool the bulk of the propellant in the tank. The goal is therefore not to simply induce propellant motion, but to suppress propellant temperature throughout the tank. Although the velocity field may appear to be fully developed, this does not insure that the propellant temperature is significantly reduced at this time in the transient. The extent of the induced motion does indicate that convection should be a fairly active process and that cooling of most of the field should be possible. Work is currently in progress to add a thermal energy equation to SOLA-ECLIPSE and thereby directly predict the relationship between fluid temperature and time.

\section{Conclusions}

The following conclusions have been drawn based on the computational analysis of flows in scale model tanks. SOLA-ECLIPSE is capable of predicting the small spread rate of laminar jets without introducing excessive artificial diffusion. The code is capable of modeling flows which involve some agitation of the free surface. The turbulence model in SOLA-ECLIPSE predicts appropriate spread rates for axisymmetric turbulent jets. Therefore, the code is judged to be a viable tool for studying jet induced mixing in cryogenic propellant tanks in a low-gravity environment.

The use of an axial jet to disperse the cooling effects of a thermodynamic vent system, and to thereby control tank pressure, appears to be a viable concept. Although actual temperature distributions have not been computed, the velocity field induced by the jet extends throughout the tank indicating that the fluid will become well mixed. Clearly, more extensive analyses are required before complete proof of concept can be claimed.

\section{References}

1. Labus, T.L., Aydelott, J.C. and Amling, G.E., "Zero-Gravity Venting of Three Refrigerants," NASA TN D-7480, 1974.

2. Aydelott, J.C., "Axial Jet Mixing of Ethanol in Cylindrical Containers During Weightlessness," NASA TP-1487, 1979.

3. Hotchkiss, R.S., "Simulation of Tank Draining Phenomena With the NASA SOLA-VOLF Code," Los Alamos Scientific Laboratory Report LA-8163MS, Dec. 1979.

4. Aydelott, J.C., "Modeling of Space Vehicle Propellant Mixing," NASA TP-2107, 1983.

5. Launder, B.E. and Spalding, D.B., Lectures in Mathematical Models of Turbulence, Academic Press, New York, 1972.

6. Launder, B.E., Morese, Ap., Rodi, W. and Spalding, B.D., "The Predication of FreeShear Flows," NASA SP-321, Vo1. 1, 1973, pp. 361-426.

7. McGuirk, J.J. and Rodi, W., "The Calculation of Three-Dimensional Free Jets," Symposium on Turbulent Shear Flows, Penn, State University, Vo1. 1, 1977, pp. 1.29-1.36.

8. Morse, A.P., "Axisymmetric Turbulent Shear Flows With and Without Swirl," Ph.D. Thesis, London University, England, 1977.

9. Pope, S.B., "An Explanation of the Turbulent Round-Jet/Plane-Jet Anomaly," AIAA Journal, Vol. 16, Mar. 1978, pp. 279-281.

10. Spalding, D.B., "Turbulence Models - A Lecture Course, Imperial College in Science and Technology CFD/82/4, 0ct. 1982.

11. Blatt, M.H., Bradshaw, R.D. and Risberg, J.A., Capillary Acquisition Devices for HighPerformance Vehicles, Executive Summary," General Dynamics/Conviar, San Diego, Ca. GDC-CRA-80-003, Feb. 1980. (NASA CR-159658). 


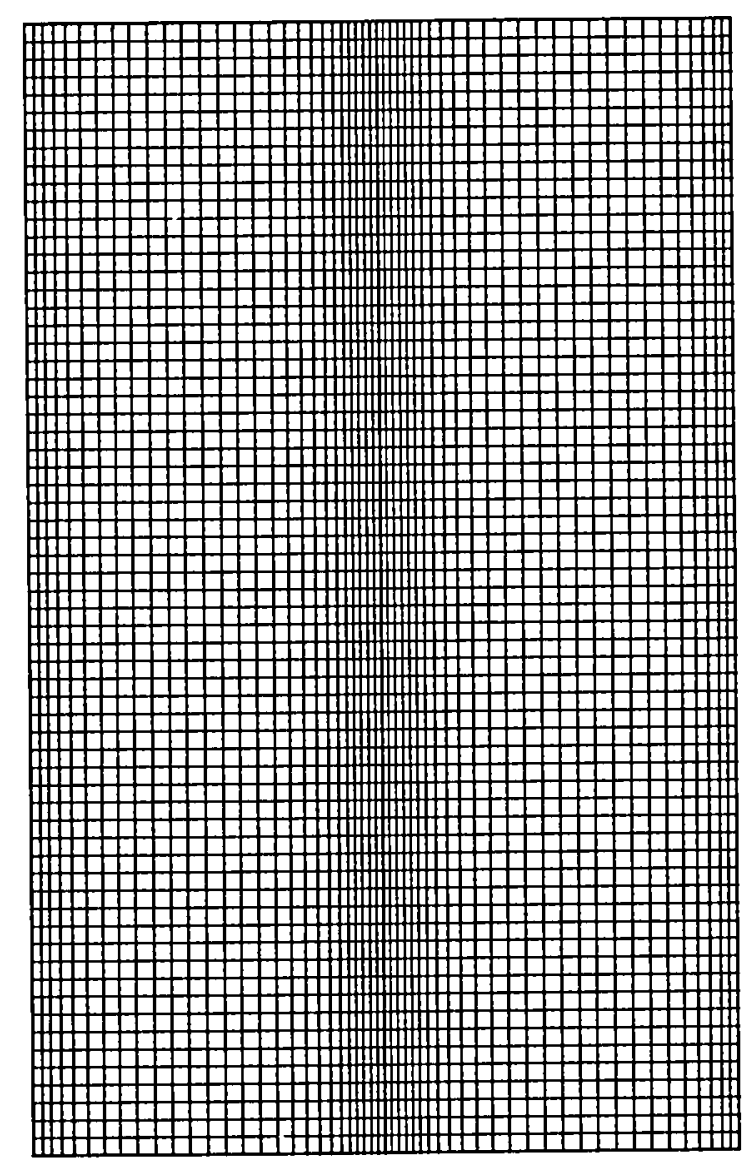

Figure 1. - Computational mesh used for analysis of scale model tanks.

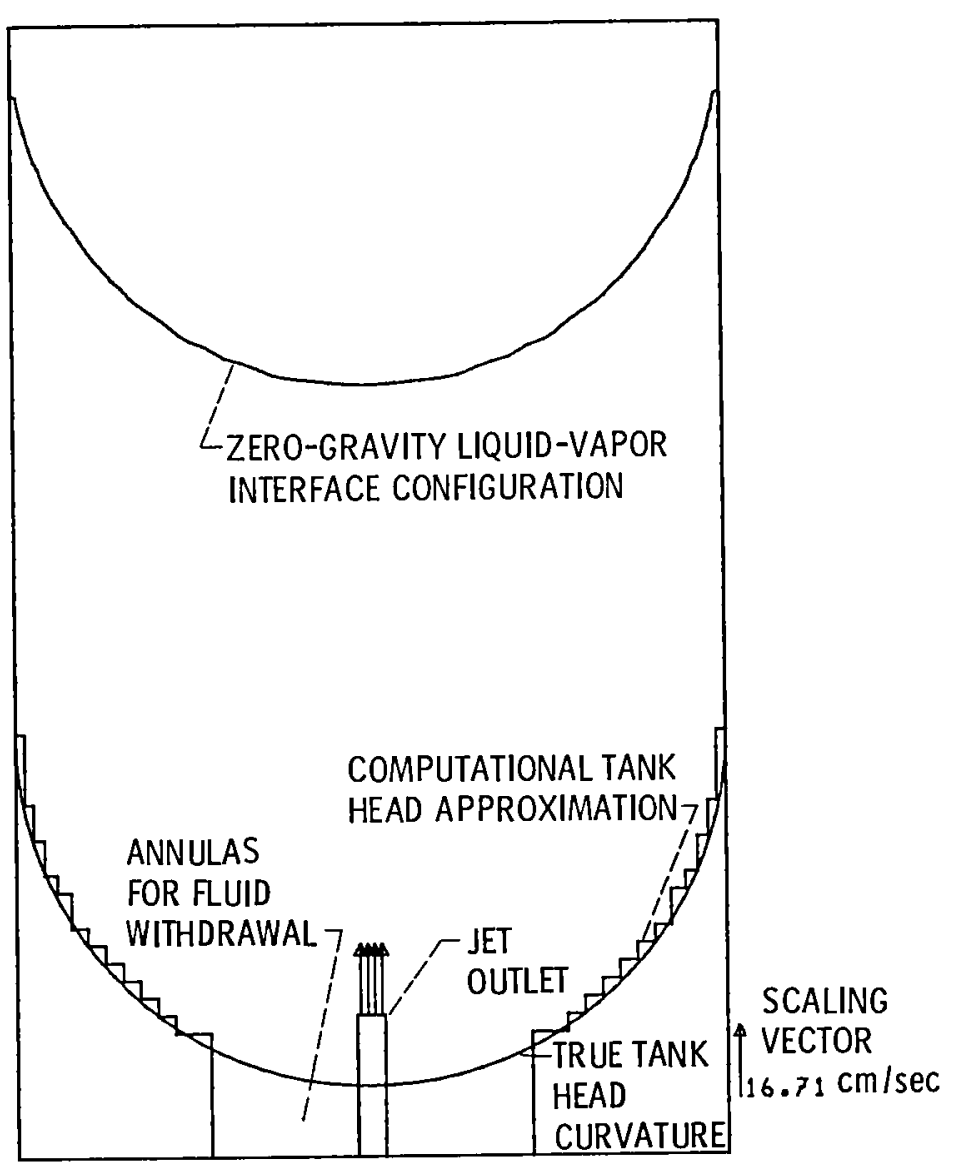

Figure 2 - Computer code approximation of scale model tank including laminar jet initial conditions. 


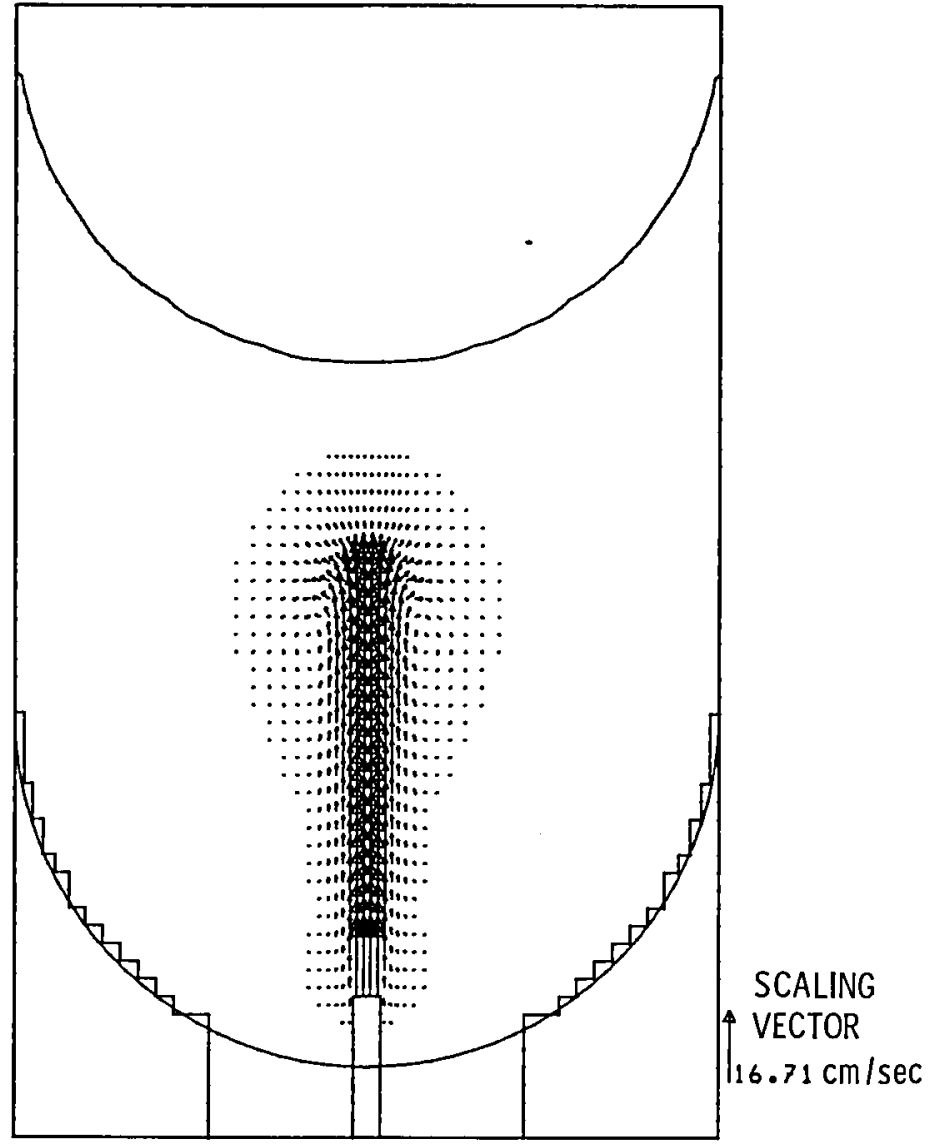

Figure 3. - Calculated velocity field with laminar jet after $1 \mathrm{sec}$ (1992 computational cycles).

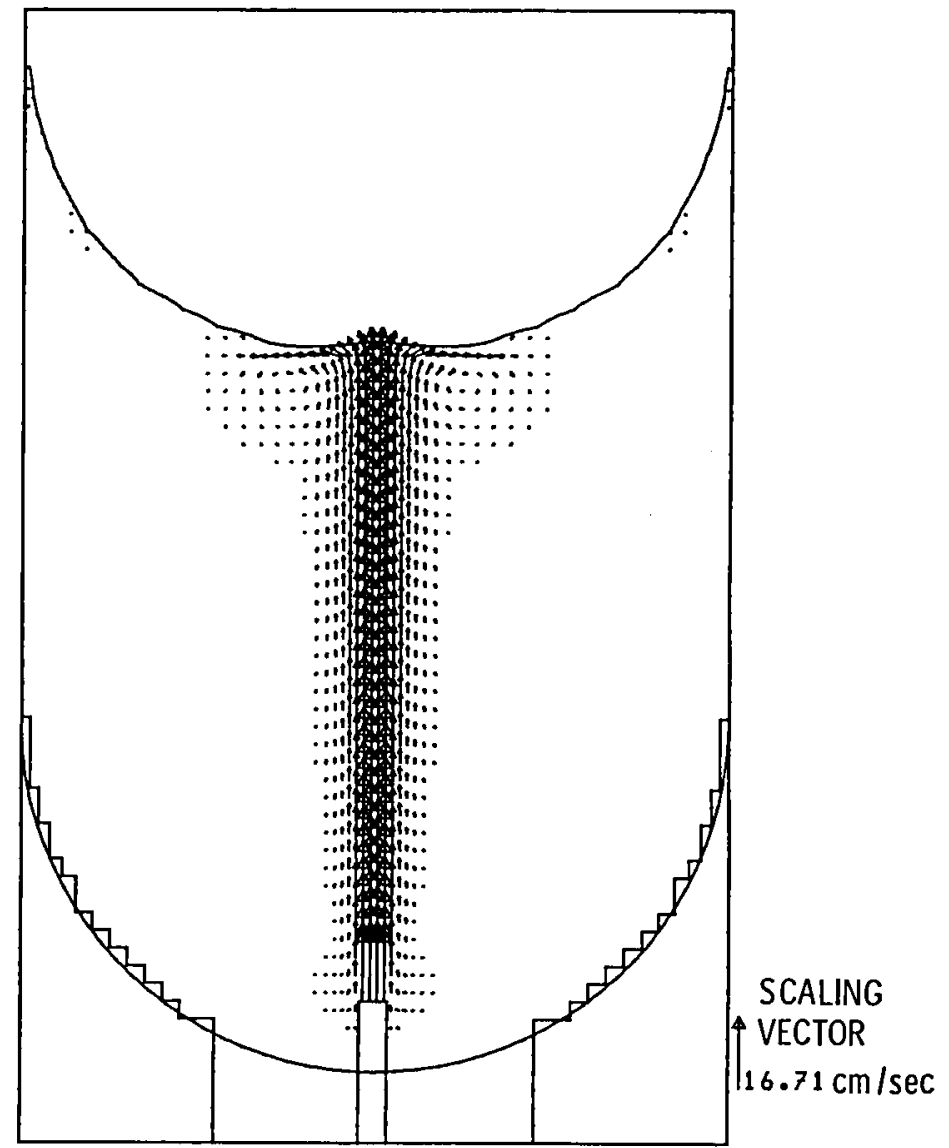

Figure 4. - Calculated velocity field with laminar jet after $2 \mathrm{sec}$ (3894 computational cycles). 


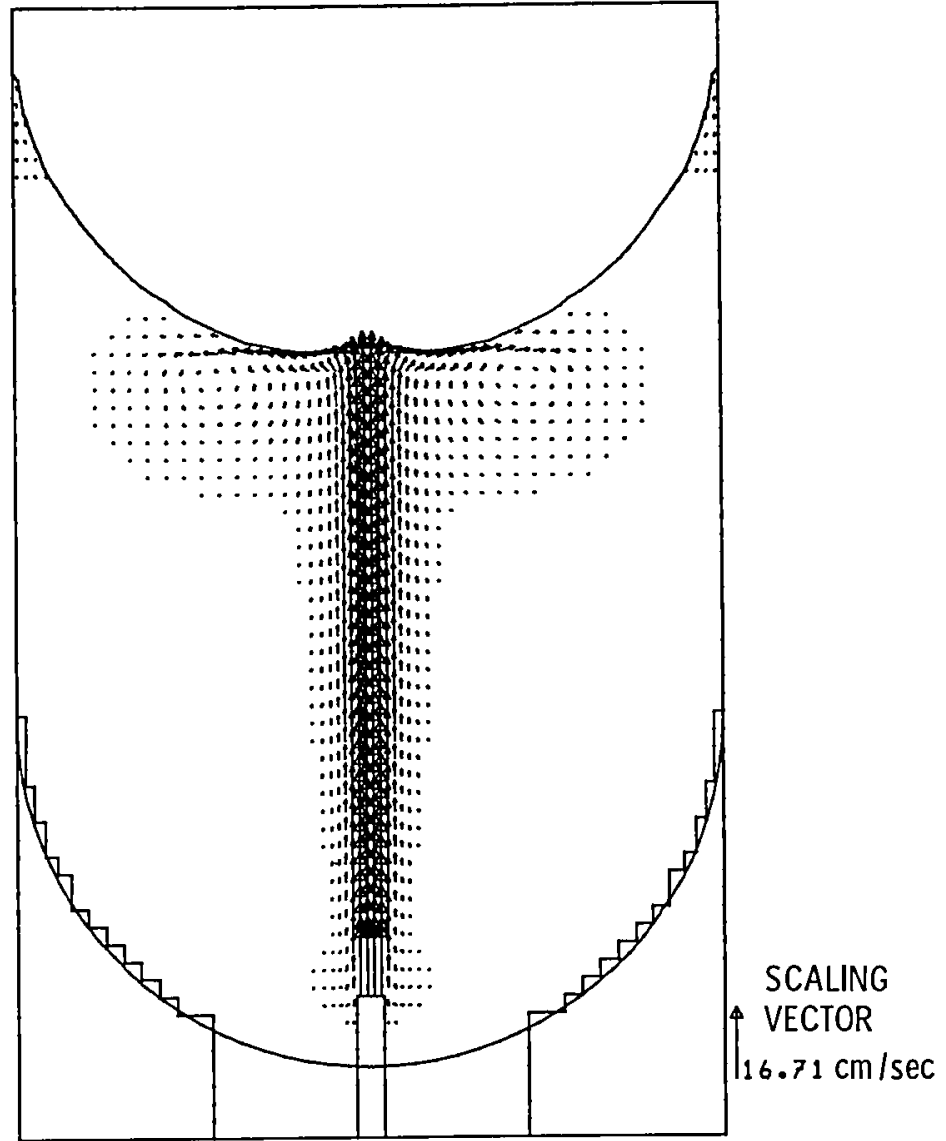

Figure 5. - Calculated velocity field with laminar jet after $3 \mathrm{sec}$ (5795 computational cycles).

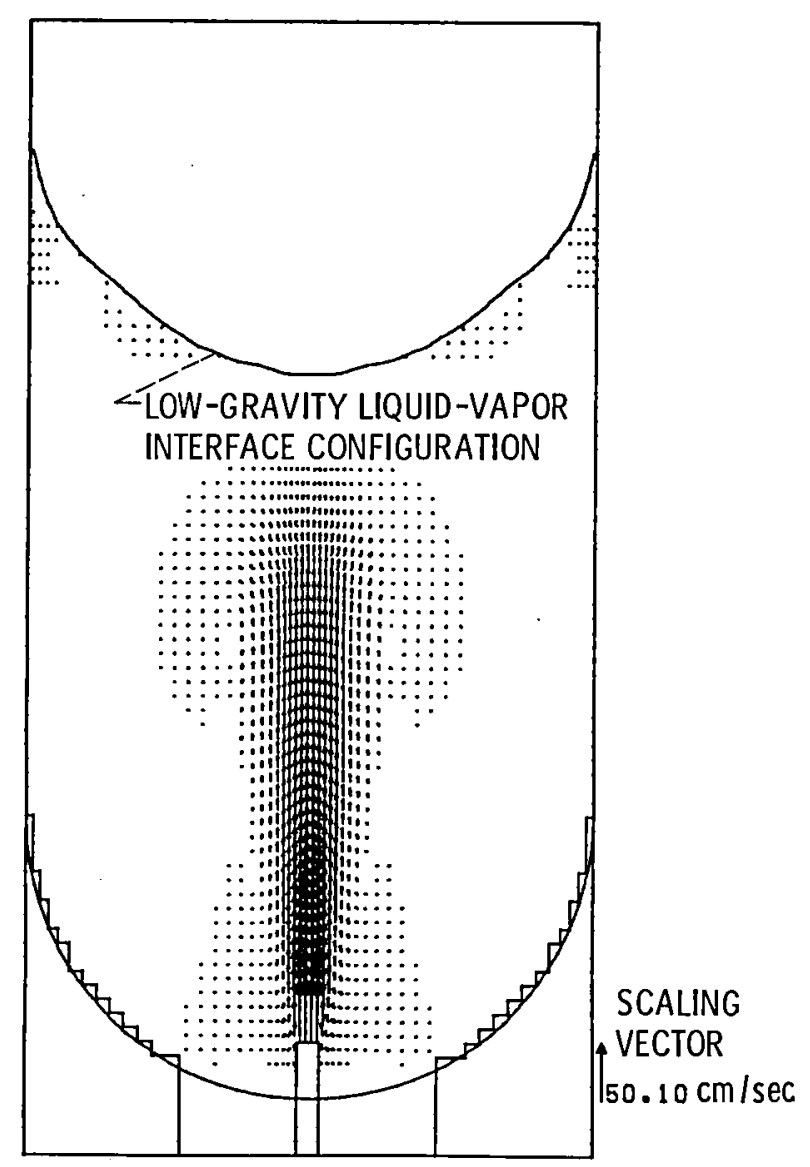

Figure 6. - Calculated velocity field with turbulent jet after $1 \mathrm{sec}$ (2086 computational cycles). 


$$
\text { I. I. }
$$




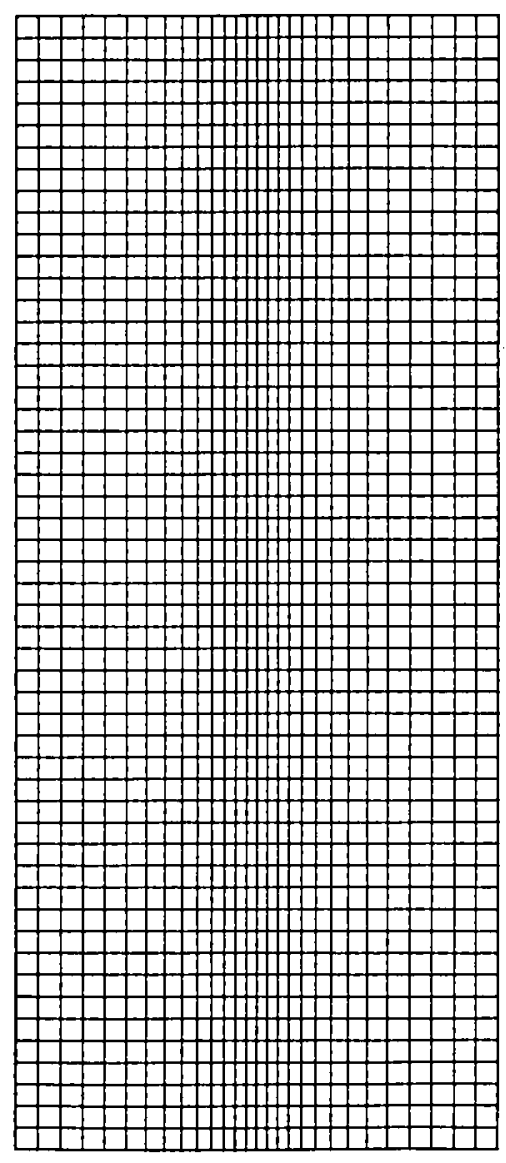

Figure 9. - Computational mesh used for analysis of full scale tanks.

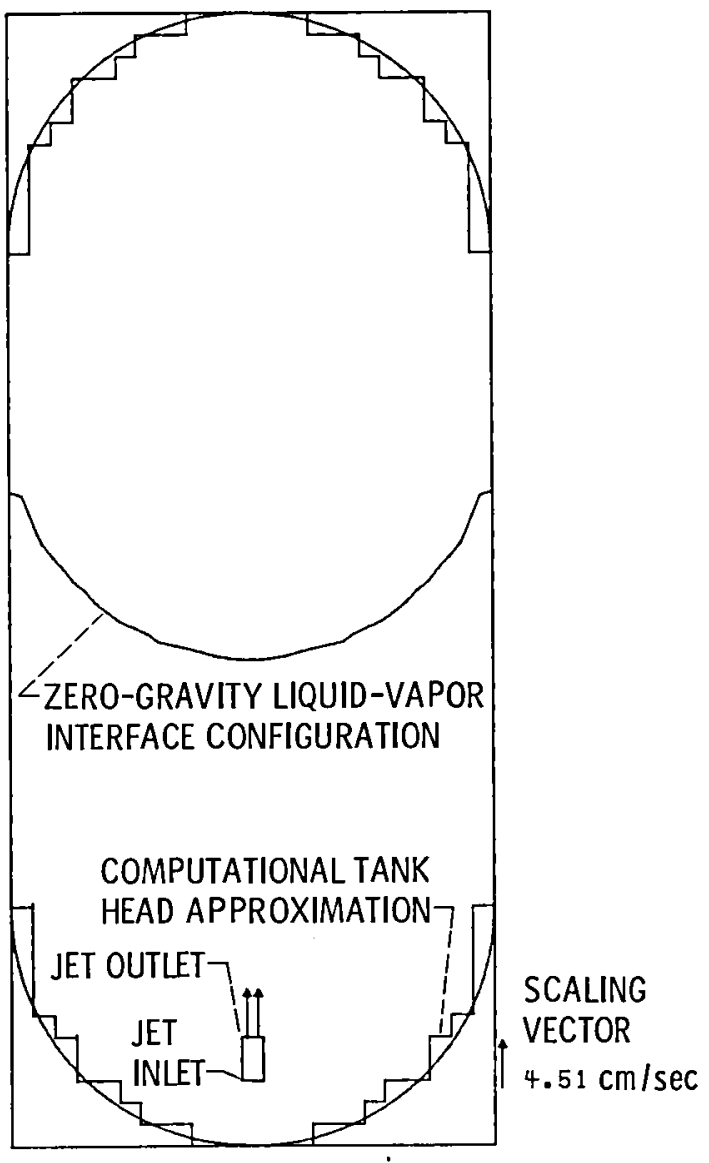

Figure 10. - Computer code approximation of full scale liquid hydrogen tank including turbulent jet initial conditions. 


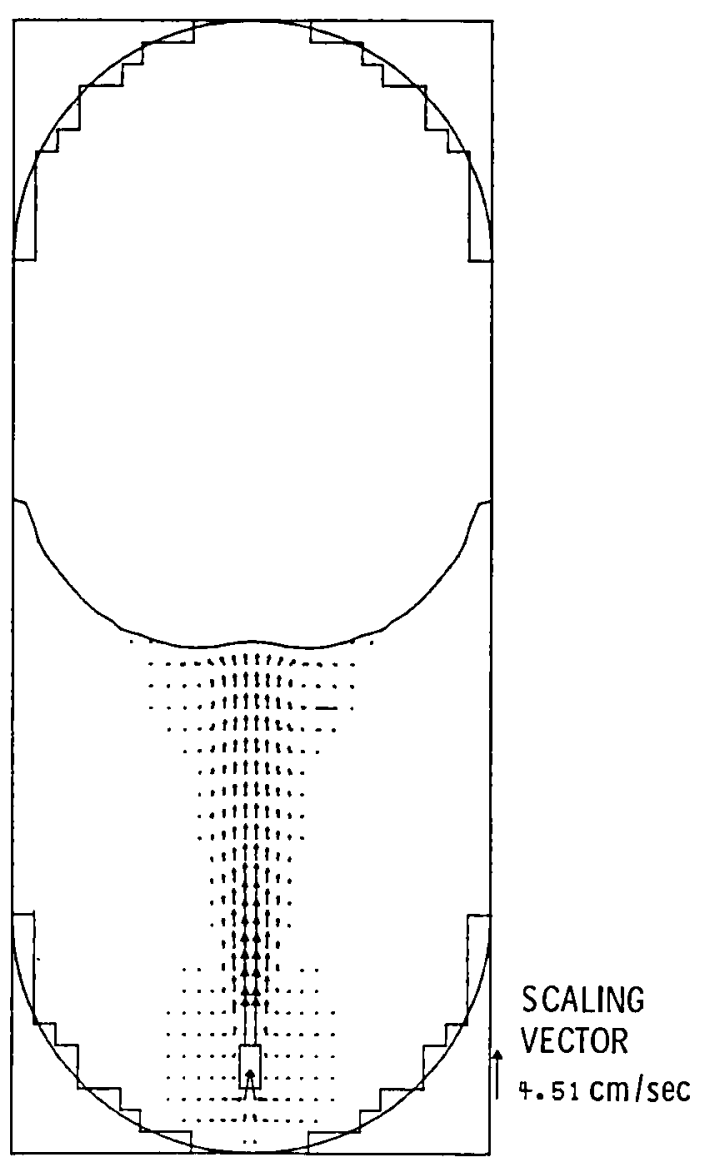

Figure 11. - Calculated velocity field for OTV liquid hydrogen tank with turbulent jet after 5 min (1315 computational cycles).

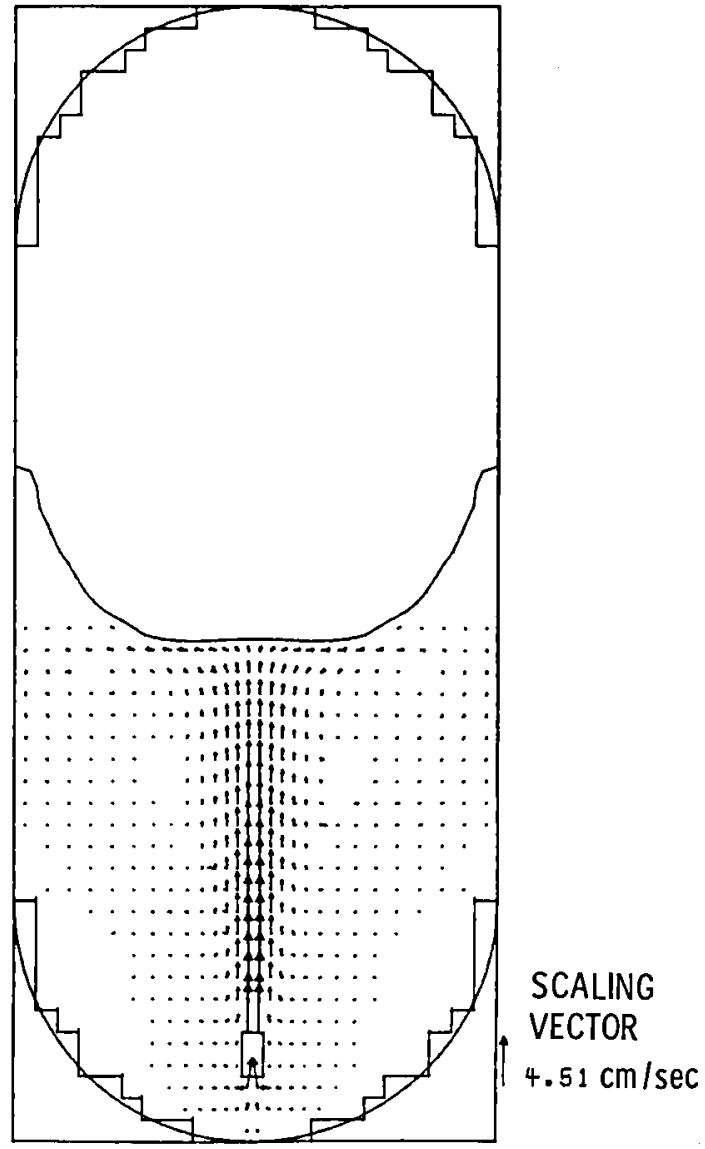

Figure 12. - Calculated velocity field for OTV liquid hydrogen tank with turbulent jet after 20 min (2952 computational cycles). 


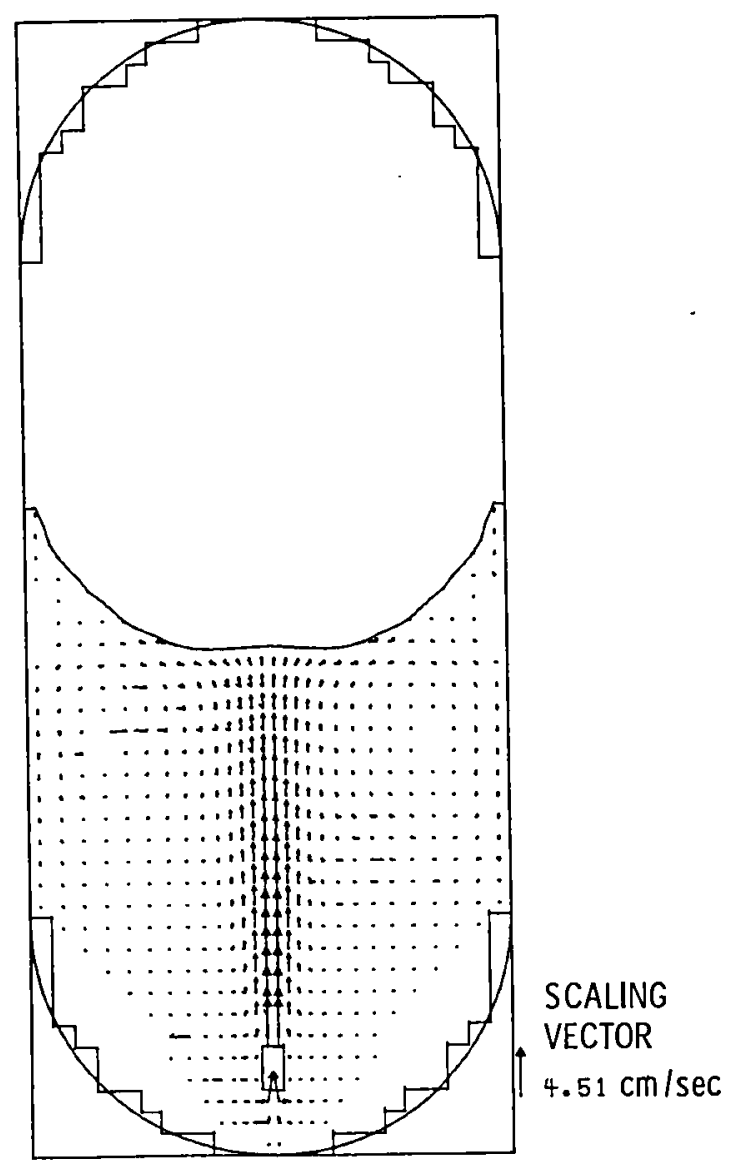

Figure 13. - Calculated velocity field for OTV liquid hydrogen tank with turbulent jet after $1 \mathrm{hr}$ (7320 computational cycles).

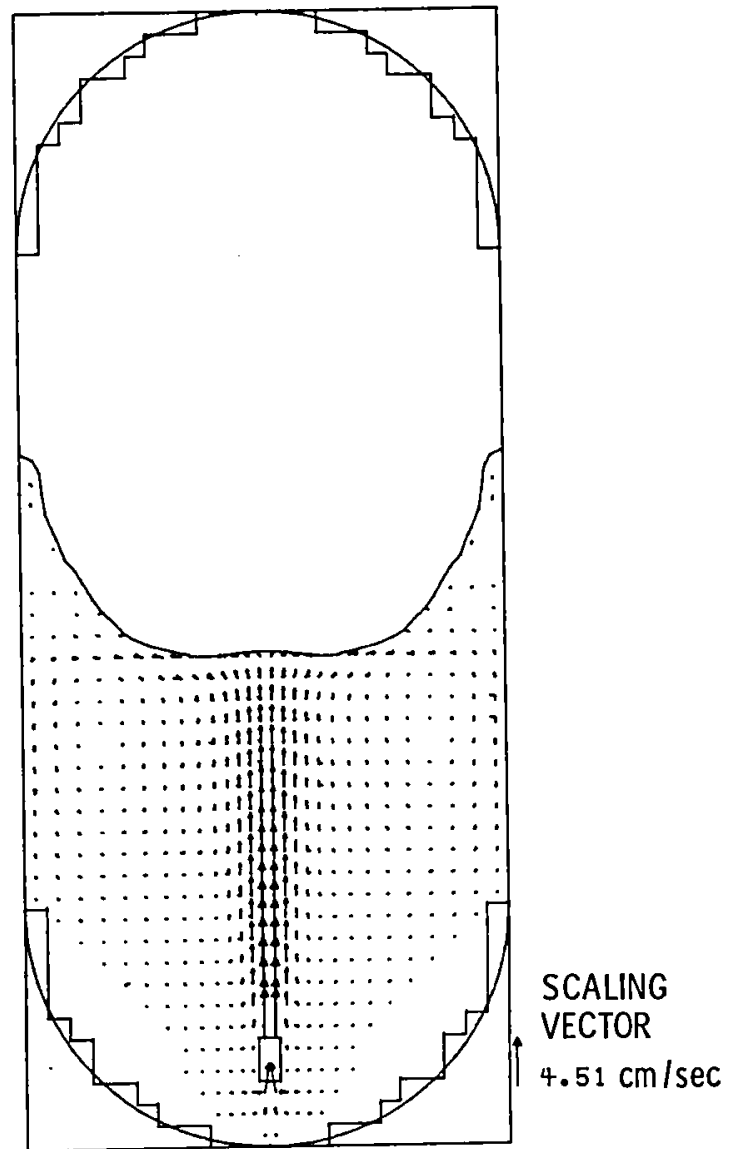

Figure 14. - Calculated velocity field for OTV liquid hydrogen tank with turbulent jet after $2 \mathrm{hr}$ (14244 computational cycles). 


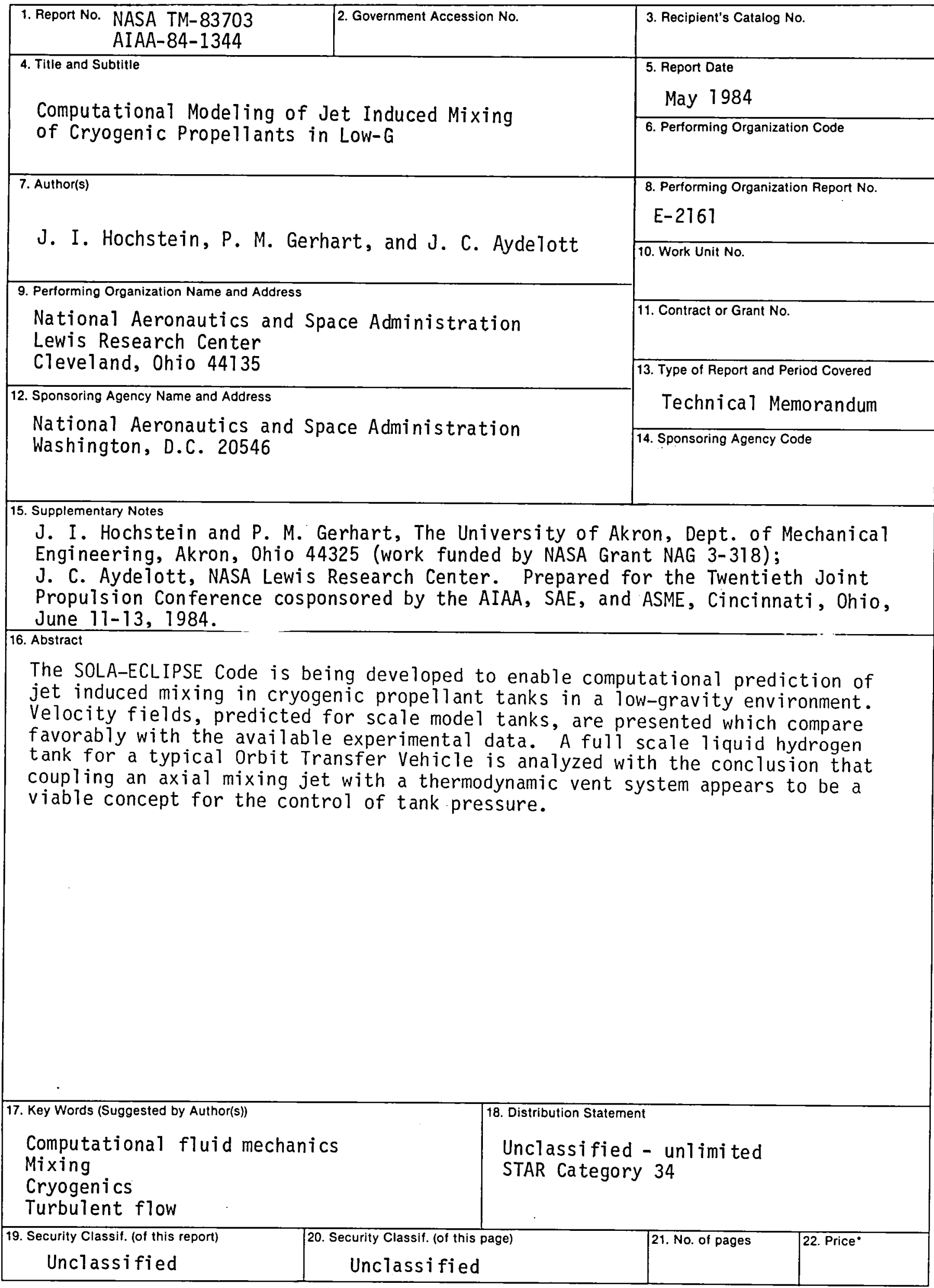

*For sale by the National Technical Information Service, Springfield, Virginia 22161 
National Aeronautics and Space Administration

Washington, D.C.

20546

Official Business

Penalty for Private Use, $\$ 300$
SPECIAL FOURTH CLASS MAIL BOOK
||||| Postage and Fees Paid
National Aeronautics and Space Administration NASA-451 